\title{
A dry gangrene signing SARS-CoV2 infection in a vulnerable patient
}

\author{
Dorsaf Elinkichari ${ }^{1}$, Faten Rabhi ${ }^{2}$, Yosra Ben Ariba ${ }^{1}$, Kahena Jaber ${ }^{1}$, and Raouf Dhaoui ${ }^{1}$ \\ ${ }^{1}$ Military Hospital of Instruction of Tunis \\ ${ }^{2}$ Hopital Militaire Principal d'Instruction de Tunis
}

December 14, 2021

\begin{abstract}
Although lungs represent the main target of the SARS-CoV2, the novel virus may affect other tissues. In the pandemic context, unexplained cutaneous ischemic lesions would be suggestive of the infection and should draw attention to a serious course of the disease.
\end{abstract}

\author{
A dry gangrene signing SARS-CoV2 infection in a vulnerable patient \\ Dorsaf Elinkichari $^{1,3}$, Faten Rabhi ${ }^{1,3}$, Yosra Ben Ariba ${ }^{2,3}$, Kahena Jaber ${ }^{1,3}$, Raouf Dhaoui ${ }^{1,3}$ \\ ${ }^{1}$ Department of Dermatology, The Military Hospital of Tunis, Tunis, Tunisia \\ ${ }^{2}$ Derpartment of Nephrology, The Military Hospital of Tunis, Tunis, Tunisia \\ ${ }^{3}$ Faculty of Medicine of Tunis, University of Tunis El Manar, Tunis, Tunisia
}

Correspondence

Dorsaf Elinkichari, Department of Dermatology, The Military Hospital of Tunis, Tunis, Tunisia.

Email:dorsaf.elinkichari@gmail.com

\section{Key Clinical message}

We believe this report will enhance our awareness of the acral ischemic signs as a predictor of the severity of the disease in COVID-19 patients.

AbstractAlthough lungs represent the main target of the SARS-CoV2, the novel virus may affect other tissues. In the pandemic context, unexplained cutaneous ischemic lesions would be suggestive of the infection and should draw attention to a serious course of the disease.

\section{Key words}

SARS-CoV2, Infection, COVID-19, Acro-ischemia, Dry gangrene

Given the patient's death, written informed consent was obtained from the patient's next of kin (Son) to publish this report in accordance with the journal's patient consent policy.

\section{Introduction}

COVID-19, the disease caused by the SARS-CoV-2, have induced an international public health emergency since its first emergence in the last months of 2019. Although lungs are the most affected organ, the infection can spread to other tissues causing multiorgan involvement. The incrimination of SARS-CoV-2 infection in 
thromboembolic complications due to hypercoagulability is now proven. However, its role in arterial diseases has been less demonstrated ${ }^{1,2}$. Cases of acute limb ischemia and different signs of acro-ischemia in infected patients have been reported ${ }^{3-6}$. We report a case of dry gangrene in a patient tested positive for SARS-CoV2 without respiratory symptoms.

\section{Observation}

A 65-year-old man with a history of hypertension, ischemic cardiomyopathy, and chronic kidney failure on dialysis presented with painful periungual erosions appeared seven days after coronarography. Upon physical examination, we noticed periungual and pulpal erosions with a fibrinous center and non-infiltrated erythematous border on both index fingers and on the left second toe (Figure 1a, 1b). Peripheral pulses were present. The ocular fundus was normal. Cutaneous biopsy was not performed since it could slow scarring. Doppler ultrasound showed signs of atherosclerosis without severe arterial obstruction. The diagnosis of cholesterol embolization syndrome was made based on clinical history and physical examination. The patient was treated as such with a good evolution after 15 days and beginning of re-epidermalization. One month later, a worsening of the same lesions was noted with the onset of dry gangrene (Figure 2), followed 48 hours later by chills and fever. He had no headache, no digestive or respiratory signs and no hypoxemia. A PCR test on nasopharyngeal swab was positive for SARS-CoV-2. Despite the initiation of anticoagulation therapy, the patient died after two days of disseminated intravascular coagulation.

\section{Discussion}

The main target of the SARS-CoV2 is the respiratory system. Typical manifestations include fever, sore throat, dry cough, fatigue, diarrhea, hyposmia and hypogeusia ${ }^{7}$. Simultaneously, the infection can be associated with multiorgan dysfunctions. The viruses bind to angiotensin-converting enzyme 2 receptors, which are present in all cells, including in endothelial cells leading to microvascular dysfunction ${ }^{8}$. Upon entering host cells, the viruses replicate and destroy them. This process induces organ damage, release of proinflammatory cytokines, chemokines, and activation of the complement system which all lead to the hyperinflammation state known as "cytokine storm" ${ }^{2}$. Both microvascular dysfunction and cytokine storm are involved in thrombotic and ischemic manifestations ${ }^{3}$. Several studies have reported different signs of acral ischemia such as pseudo-chilblains, livedo reticularis and dry gangrene $\mathrm{e}^{4-6}$. Chilblains occur more commonly in young patients with mild or asymptomatic form of the disease ${ }^{4,6}$. However, as in our case, the other manifestations of acral ischemia, including gangrene and livedo reticularis often occur in patients with severe disease, with a mortality rate of $10 \%{ }^{6}$. This group of patients may present with a misleading form of the disease in a context of a confusing hidden severity. Thus, they don't always develop signs that require hospitalization, like the case of our patient who did not develop respiratory signs or asymptomatic hypoxemia.

The particularity of our case consists in the appearance of ischemic skin manifestations of SARS-CoV2 infection and in the severe course of the disease leading to death without any respiratory signs. Considering the arteriopathy and the cholesterol embolization syndrome, the infection precipitated the evolution of lesions that would heal towards dry gangrene.

\section{Conclusion}

In the pandemic context, the appearance of unexplained cutaneous ischemic signs, even if isolated, would be suggestive of the infection. Acro-ischemic signs may indicate a state of severe systemic hypercoagulability and should draw attention to a serious course of the disease.

\section{CONFLICT OF INTEREST}

None. This research received no specific grant from any funding agency in the public, commercial, or notfor-profit sectors.

\section{AUTHOR CONTRIBUTIONS}

D. Elinkichari is the guarantor of the content of the manuscript, including the data and analysis. F. Rabhi contributed to acquisition of data, conception, and interpretation of information, revised it critically for im- 
portant intellectual content, and gave final approval of the version to be submitted. Y. Ben Ariba contributed to acquisition and analysis of data. K. Jaber revised data critically for important intellectual content. MR. Dhaoui contributed to interpretation of data and revision of the manuscript.

\section{ETHICAL STATEMENT}

Written informed consent was obtained from the patient's next of kin (Son).

\section{References}

1. Ward A, Sarraju A, Lee D, et al. COVID-19 is associated with higher risk of venous thrombosis, but not arterial thrombosis, compared with influenza: Insights from a large US cohort. medRxiv. Published online October 18, 2021:2021.10.15.21264137. doi:10.1101/2021.10.15.21264137

2. Loo J, Spittle DA, Newnham M. COVID-19, immunothrombosis and venous thromboembolism: biological mechanisms. Thorax . 2021;76(4):412-420. doi:10.1136/thoraxjnl-2020-216243

3. Santosa YP, Yuwono A. Two Different Clinical Presentations of Acute Limb Ischemia Caused by Acute Thrombotic Events in COVID-19.Cureus . 13(9):e17916. doi:10.7759/cureus.17916

4. Alramthan A, Aldaraji W. Two cases of COVID-19 presenting with a clinical picture resembling chilblains: first report from the Middle East. Clin Exp Dermatol . 2020;45(6):746-748. doi:10.1111/ced.14243

5. Bouaziz JD, Duong TA, Jachiet M, et al. Vascular skin symptoms in COVID-19: a French observational study. J Eur Acad Dermatol Venereol . 2020;34(9). doi:10.1111/jdv.16544

6. Galván Casas C, Català A, Carretero Hernández G, et al. Classification of the cutaneous manifestations of COVID -19: a rapid prospective nationwide consensus study in Spain with 375 cases. Br J Dermatol . 2020;183(1):71-77. doi:10.1111/bjd.19163

7. Azer SA. COVID-19: pathophysiology, diagnosis, complications and investigational therapeutics. New Microbes and New Infections . 2020;37:100738. doi:10.1016/j.nmni.2020.100738

8. Becker RC. COVID-19 update: Covid-19-associated coagulopathy. J Thromb Thrombolysis . Published online May 15, 2020:1-14. doi:10.1007/s11239-020-02134-3

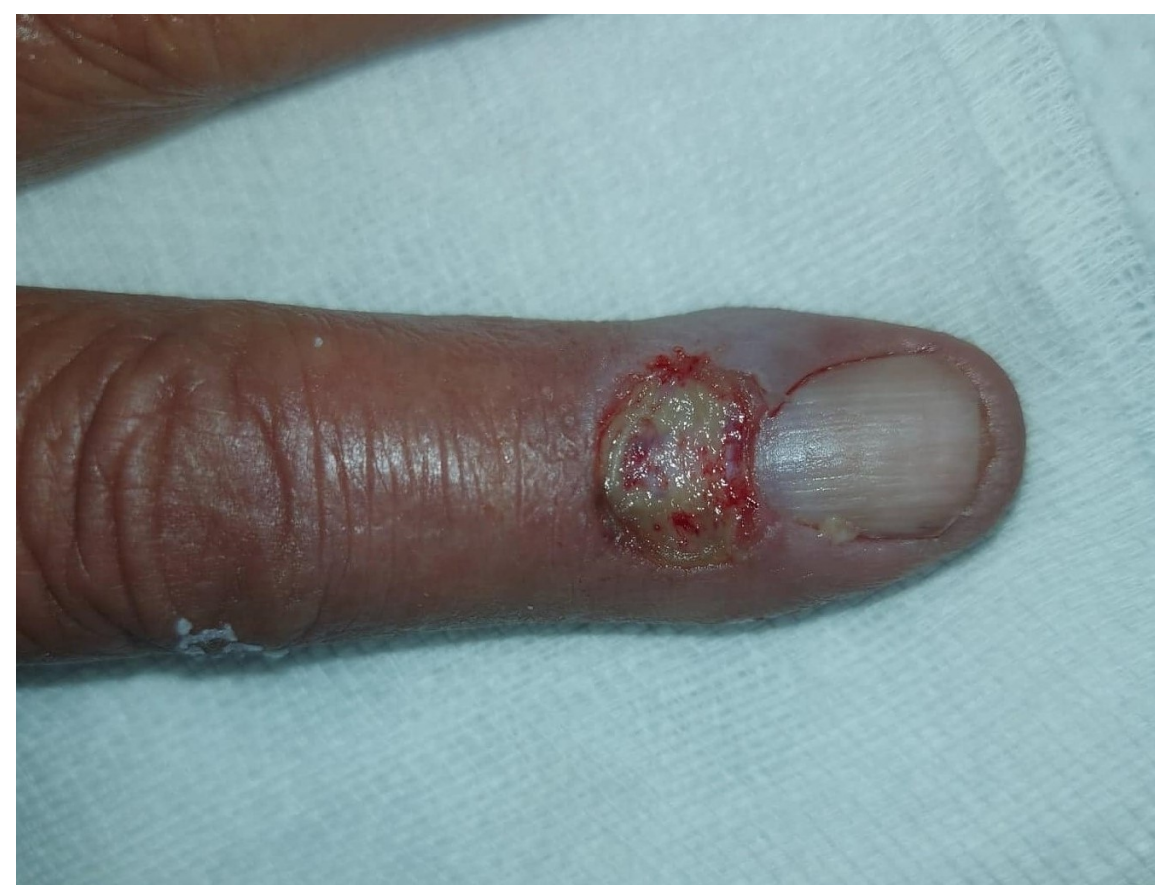





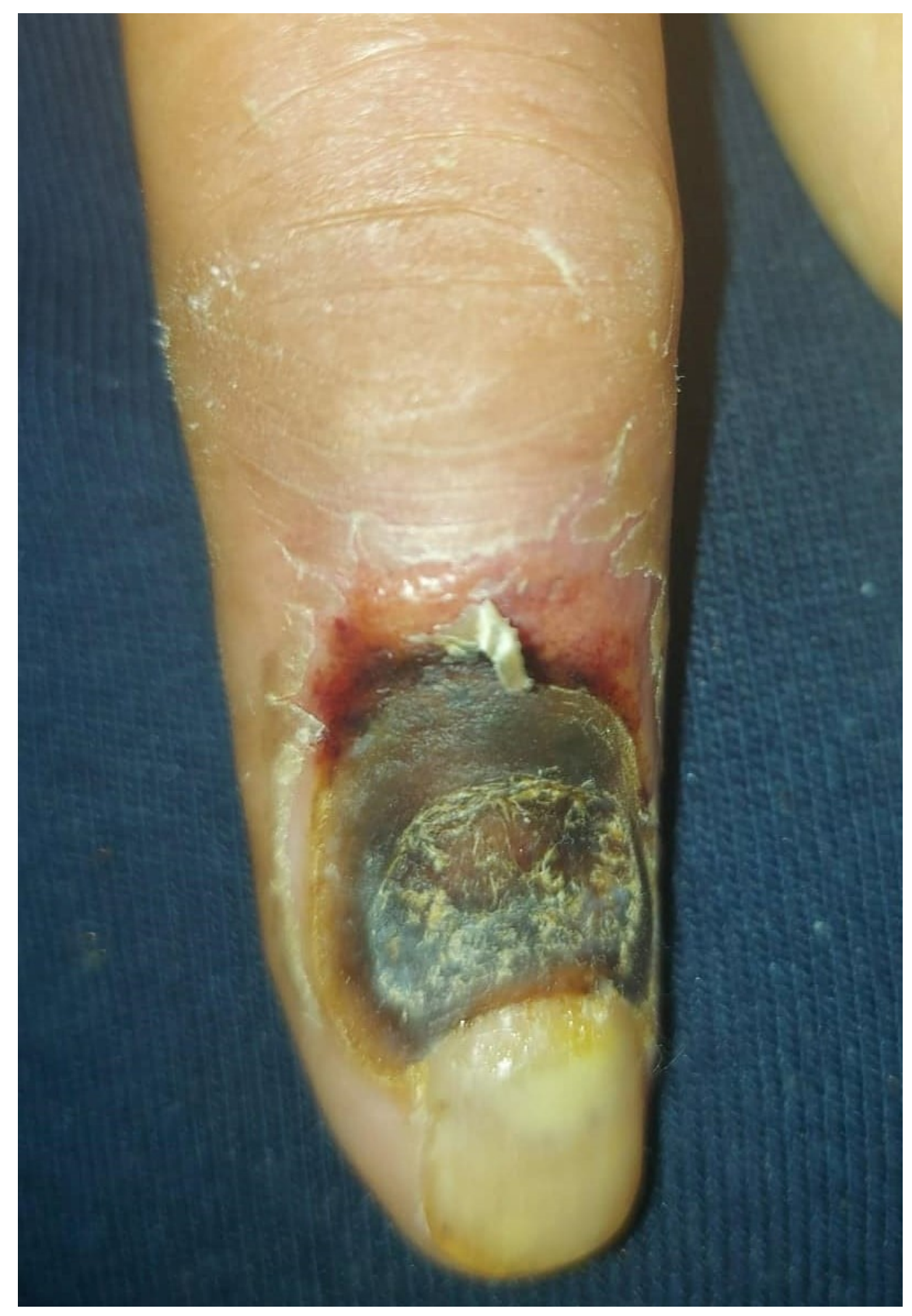

